\title{
ESTRUTURA DO LENHO DE Plathymenia reticulata E ALGUMAS IMPLICAÇÕES NA EFICIÊNCIA HIDRÁULICA E RESISTÊNCIA MECÂNICA
}

\author{
Eduardo Luiz Longui ${ }^{1}$, Israel Luiz de Lima $^{2}$, Sandra Monteiro Borges Florsheim ${ }^{1}$, \\ Antônio Carlos Galvão de $\mathrm{Melo}^{3}$, Diego Romeiro ${ }^{4}$, Itiberê Moura Sckerratt Suckow ${ }^{5}$, \\ Luana Naves Testoni ${ }^{4}$
}

${ }^{1}$ Biólogo, Dr., Seção de Madeira e Produtos Florestais, Instituto Florestal, Horto Florestal, São Paulo, SP, Brasil - elongui @if.sp.gov.br; sflorsheim@if.sp.gov.br

${ }^{2}$ Eng. Florestal, Dr., Seção de Madeira e Produtos Florestais, Instituto Florestal, São Paulo, SP, Brasil - israelluizde.lima@ yahoo.com.br ${ }^{3}$ Eng. Florestal, Dr., Floresta Estadual de Assis, Assis, SP, Brasil - acgmelo@ gmail.com

${ }^{4}$ Biólogo, Instituto Florestal, São Paulo, SP, Brasil - diego.romeiro@gmail.com; lua_bio1@yahoo.com.br

${ }^{5}$ Graduando em Engenharia Ambiental, Faculdades Oswaldo Cruz, São Paulo, SP, Brasil - itibere.suckow@gmail.com

Recebido para publicação: 24/10/2010 - Aceito para publicação: 06/12/2011

\section{Resumo}

Investigou-se a variação anatômica, velocidade de propagação do som (v), densidade aparente $\left(\rho_{\text {ap }}\right)$ e módulo de elasticidade dinâmico (MOEd) na madeira de Plathymenia reticulata. Verificou-se como as variações nessas características podem influenciar na eficiência hidráulica e resistência mecânica. Para a anatomia, empregaram-se as metodologias usuais, a (v) foi medida com equipamento de ultrassom, a $\left(\rho_{\mathrm{ap}}\right)$ foi determinada pelo método da balança hidrostática, e a partir dos valores de (v) e $\left(\rho_{\text {ap }}\right)$ foi calculado o (MOEd). O diâmetro de vasos aumentou na direção da casca, o que deve acarretar melhor eficiência hidráulica. A frequência de vasos e o MOEd correlacionaram-se negativamente na região da medula, indicando madeira mais flexível e mais resistente à ação dos ventos. Raios mais frequentes diminuem a (v), e como esta também indica a rigidez, regiões com maior frequência de raios apresentam maior flexibilidade. Fibras com paredes mais espessas na base do fuste podem aumentar a resistência das árvores ao receberem uma carga extra. A relação negativa entre $\rho_{a p}$ e lume das fibras deve-se ao fato de o lume representar espaços vazios que não contribuem na massa. Raios mais largos na direção da casca podem auxiliar no armazenamento de amido, que, quando hidrolisado, também atua na recuperação de vasos embolizados.

Palavras-chave: Amarelinho; anatomia da madeira; propriedades da madeira; variação axial; variação radial.

\begin{abstract}
Wood structure of Plathymenia reticulata and some implications on hydraulic efficiency and mechanical strength. We investigated the anatomical variation, speed of sound propagation (v), specific gravity $\left(\rho_{\mathrm{ap}}\right)$ and dynamic modulus of elasticity (MOEd) in the wood of Plathymenia reticulate Benth. The aim was to determine how variations in these characteristics may influence the hydraulic efficiency and mechanical strength. The anatomical features were analyzed according to usual techniques; (v) was measured with ultrasound equipment, $\left(\rho_{\mathrm{ap}}\right)$ was determined with "balança hidrostática" method and from (v) and $\left(\rho_{\text {ap }}\right)$ values was calculated (MOEd). The results showed that increase in vessel diameter from pith to bark can result in better hydraulic efficiency. The negative correlation between vessels frequency and MOEd in the pith indicates more flexible wood, contributing to the resistance of young tree to wind action. Rays more frequent decreased (v), as this is also an indication of stiffness; regions with the highest ray frequency provide higher flexibility. Fibers with thicker walls at the base of the stem can increase the tree resistance when they receive an extra load. The negative relationship between $\rho_{\text {ap }}$ and fibers lumen is due increase in empty spaces that no contributed in wood mass. Wider rays in the bark direction can contributed in the storage of starch, which when hydrolyzed can retrieve embolized vessels.
\end{abstract}

Keywords: Amarelinho; wood anatomy; wood properties; axial variation; radial variation. 


\section{INTRODUÇÃO}

O amarelinho, Plathymenia reticulata Benth. (Fabaceae), é uma espécie arbórea de ocorrência muito ampla no Brasil, desde o Amapá até o Paraná, sendo encontrada na Mata Atlântica, no Cerrado, na Caatinga e no Pantanal. Apresenta crescimento rápido e chega a atingir altura total de $20 \mathrm{~m}$, com diâmetro à altura do peito de $80 \mathrm{~cm}$. A madeira de $P$. reticulata, serrada ou roliça, tem ampla utilização para móveis, painéis, portas, construção naval, acabamentos internos, tonéis, postes, carrocerias, estacas, esteios e mourões (CARVALHO, 2008). Jenrich (1989) relata que é bastante durável mesmo para utilização em mourões de cerca, por ser resistente a cupins.

Não foram encontrados estudos que caracterizassem a madeira dessa espécie no sentido radial e axial, além de investigar relações da anatomia da madeira com a eficiência hidráulica e a resistência mecânica. Tal fato, de acordo com Woodrum et al. (2003), deve-se à complexidade das relações entre a anatomia e aquelas duas funções das células do tronco, sendo poucos os estudos que abordaram essas três variáveis conjuntamente; o mais comum é encontrar trabalhos que enfoquem apenas as duas primeiras.

Contudo, entende-se que os padrões ecológicos na anatomia do xilema devem ser discutidos em termos de seus significados funcionais, pois, para uma compreensão total da fisiologia das árvores, é necessário considerar as várias funções da madeira da raiz, caule e ramos simultaneamente e tentar desvendar as relações causais entre, por exemplo, o funcionamento hidráulico e a resistência mecânica.

Muitos autores, como Wagner et al. (1998), propõem o estudo da madeira em um contexto de "tradeoff", termo muito usado em Economia (SANDRONI, 2002), que pode ser entendido da seguinte forma: quanto mais se investe em uma qualidade ou característica, perde-se em outra qualidade ou característica. Assim, mais do que uma questão de otimizações simples, as adaptações para um tipo de resposta ao ambiente afetam como as plantas se adaptam a outro tipo de estresse (CHRISTENSENDALSGAARD et al., 2008). Um exemplo extremo seria uma madeira com maximização na eficiência hidráulica, com muitos vasos de grande diâmetro, o que sem dúvida aumentaria o transporte de água, no entanto, seria tão fraca mecanicamente a ponto de se quebrar antes de florescer e produzir sementes para uma nova geração (BAAS et al., 2004).

Por meio do conhecimento da variação anatômica radial e axial da madeira de Plathymenia reticulata e de propriedades como sua velocidade de propagação do som, densidade aparente e módulo de elasticidade dinâmico, objetivou-se verificar como as variações anatômicas podem influenciar na eficiência hidráulica e resistência mecânica dessas árvores.

\section{MATERIAL E MÉTODOS}

Para este estudo, foram avaliadas três árvores de Plathymenia reticulata. Do fuste de cada árvore foram retirados três discos da região da base, meio e topo, totalizando nove discos (Tabela 1). As árvores abatidas foram plantadas em reflorestamento puro da espécie, em espaçamento de $3 \times 2 \mathrm{~m}$, e na ocasião do corte tinham 19 anos. O material foi coletado na Floresta Estadual de Assis (FEA), localizada no município de Assis, São Paulo. Na FEA ocorre naturalmente cerradão (fisionomia floresta de cerrado) e o clima é do tipo Cwa, quente com inverno seco, temperatura média anual de $20^{\circ} \mathrm{C}$ e precipitação média anual de $1400 \mathrm{~mm}$ (MAX et al., 2007). A figura 1 apresenta uma caracterização climática do município de Assis com base nos dados do Centro de Pesquisas Meteorológicas e Climáticas Aplicadas à Agricultura (CEPAGRI, 2010). Uma amostra do lenho de cada árvore foi depositada na Xiloteca do Instituto Florestal (SPSFw).

Tabela 1. Informações sobre as árvores de Plathymenia reticulata.

Table 1. Information of the Plathymenia reticulata trees.

\begin{tabular}{lcccc}
\hline $\mathbf{N}^{\circ}$ Xiloteca (SPSF w) & Altura (m) & Diâmetro base (cm) & Diâmetro meio (cm) & Diâmetro topo (cm) \\
\hline 3708 & 11,6 & 24,0 & 19,5 & 17,5 \\
3709 & 11,9 & 22,5 & 16,0 & 14,0 \\
3710 & 10,4 & 21,0 & 19,0 & 17,0 \\
\hline
\end{tabular}

Dos discos com cerca de $6 \mathrm{~cm}$ de espessura, foram desdobradas tiras radiais, das quais retiraramse amostras em quatro posições no sentido medula-casca $(0=$ medula; 1 = intermediária 1 ; 2 = intermediária 2; e 3 = casca), totalizando 36 amostras (Figura 2). Inicialmente, as amostras tiveram a velocidade de propagação do som determinada empregando-se o equipamento de ultrassom G. Lucchi 
Elasticity Tester, o qual produz um impulso ultrassônico e possui duas sondas, uma geradora e outra receptora do sinal. Para obtenção da velocidade de propagação do som, as sondas foram posicionadas nas extremidades de cada amostra, anotando-se o tempo que o impulso levou para percorrer o corpo-deprova. Em seguida, calculou-se a velocidade de propagação do som com a seguinte expressão: $\mathrm{v}=$ metros/segundo, conforme descrito por Lucchi (1986), sendo essa análise essencial para o cálculo do módulo de elasticidade dinâmico.

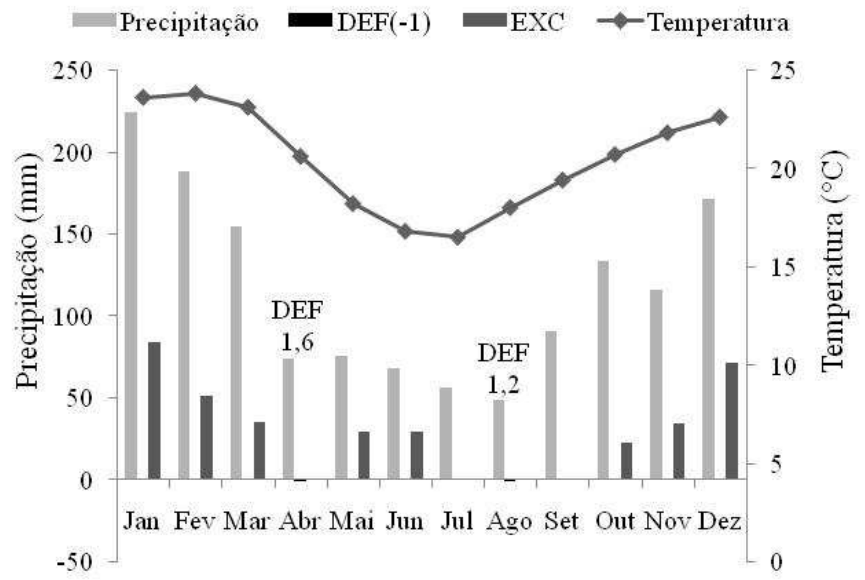

Figura 1. Caracterização climática do município de Assis onde se localiza a Floresta Estadual de Assis (FEA). Variação anual da precipitação e temperatura: extrato do balanço hídrico mensal. Período de 1961-1990 (CEPAGRI, 2010).

Figure 1. Climate characterization of the Assis, city which is located the State Forest of Assis (FEA). Annual variation in precipitation and temperature: Monthly hydric balance. Period 1961-1990 (CEPAGRI, 2010).

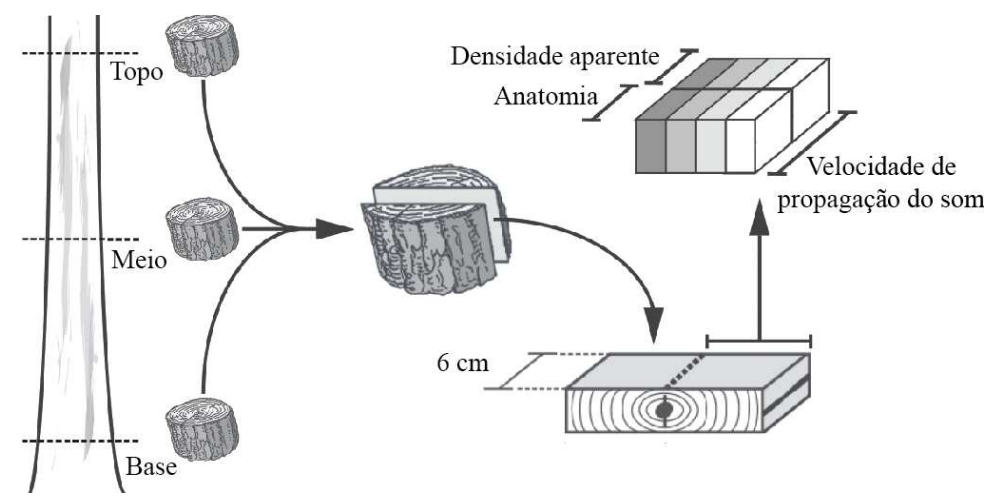

Figura 2. Representação esquemática da amostragem para as análises anatômicas, densidade aparente e velocidade de propagação do som.

Figure 2. Schematic representation of samples for anatomical features, specific gravity and speed of sound propagation.

Em seguida, as amostras foram cortadas ao meio, para determinação da densidade aparente e análises anatômicas. Para a densidade aparente, utilizou-se o método da balança hidrostática, segundo Silveira et al. (1999). Com os valores obtidos, foi empregada a expressão:

$$
\rho_{a p}=\frac{P_{u}}{V_{u}}
$$


Em que: $\rho_{\text {ap }}=$ densidade aparente $\left(\mathrm{kg} \cdot \mathrm{m}^{-3}\right)$;

$\mathrm{P}_{\mathrm{u}}=$ massa do corpo-de-prova, a $15 \%$ de umidade $(\mathrm{kg})$;

$\mathrm{V}_{\mathrm{u}}=$ volume deslocado pelo do corpo-de-prova, $15 \%$ de umidade $\left(\mathrm{m}^{3}\right)$.

A partir dos valores da velocidade de propagação do som através da madeira e da densidade, foi calculado o módulo de elasticidade dinâmico $\left(\mathrm{MOE}_{\mathrm{d}}\right)$, conforme Lucchi (1986), empregando-se a seguinte expressão:

$$
\text { MOEd }=\frac{v^{2}}{100} \times \rho \text { ap }
$$

Em que: $M O E_{d}=$ módulo de elasticidade dinâmico (MPa);

$v=$ velocidade de propagação do som $\left(\mathrm{m}^{-1} \mathrm{~s}^{-1}\right)$

$\rho_{\text {ap }}=$ densidade aparente $\left(\mathrm{kg} \cdot \mathrm{m}^{-3}\right)$.

Para as análises anatômicas, de cada corpo-de-prova retiraram-se fragmentos para o estudo das células dissociadas (BERLYN; MIKSCHE, 1976). Em seguida, os corpos-de-prova foram amolecidos por meio do cozimento em água e glicerina na proporção de (4:1) até mostrarem condições ideais para o seccionamento. Secções histológicas nos planos transversal, longitudinal tangencial e longitudinal radial foram obtidas em micrótomo de deslize Zeiss-Hyrax S50, com espessura entre 14 e $20 \mu \mathrm{m}$, sendo as mesmas processadas de acordo com Sass (1951). As análises seguiram as recomendações do IAWA Committee (1989). Todas as mensurações foram realizadas em microscópio Olympus modelo CX 31, equipado com computador e software de análise de imagens Image-Pro Plus 6.3.

Os resultados foram avaliados por meio de análise de variância paramétrica (One Way Analysis of Variance), e quando uma diferença significativa foi observada, aplicou-se teste de comparações múltiplas (Tukey) para identificar os pares determinantes das diferenças. Também foi realizada análise de correlação de Pearson, para determinar as relações entre as características anatômicas e as propriedades estudadas.

\section{RESULTADOS}

Os resultados são apresentados separadamente por tipo de célula. Não foram realizadas comparações entre as posições axiais, uma vez que os discos apresentavam diâmetros diferentes e possuíam números distintos de camadas de crescimento. Dessa forma, uma análise axial compararia amostras que representariam diferentes idades do câmbio.

\section{Vasos}

O diâmetro dos vasos apresentou diferenças significativas entre as posições radiais, em todas as alturas. Os maiores valores ocorreram na região da casca, sendo que no meio e topo do tronco há aumento no diâmetro em direção da casca. Já na base ocorreu aumento nas duas primeiras posições, seguido de redução para a posição intermediária 2, com aumento para a casca (Figura 3a). O comprimento dos elementos de vaso mostrou variações distintas conforme a altura estudada. Na base, o menor valor foi encontrado na medula; já no meio e topo do tronco ocorreu uma queda na região intermediária 1 , seguida de aumento no sentido da casca, que não diferiu dos valores encontrados na medula (Figura 3b). Para a frequência dos vasos, nas três alturas os maiores valores ocorreram na medula (Figura 3c).

\section{Raios}

A altura dos raios em micrometros apresentou variação significativa apenas no meio do tronco, sendo detectado aumento em direção da casca (Figura 4a). Para a largura dos raios em micrometros, foram notadas variações em todas as alturas, com aumento dos valores na direção da casca, com exceção da base, que mostrou uma ligeira queda da posição intermediária 2 (2) para a casca (3), embora as duas não tenham diferido estatisticamente (Figura $4 \mathrm{~b}$ ). A frequência dos raios diminuiu significativamente em direção da casca, na base e no topo do tronco, diferente do meio do tronco, que mostrou oscilação dos valores, com as maiores frequências de raios na casca (Figura 4c).

\section{Fibras}

Mesmo com algumas oscilações, o comprimento aumentou significativamente na direção da casca nas três alturas (Figura 6a). O diâmetro das fibras também aumentou no sentido da casca na base e 
topo do tronco, contudo não apresentou variação radial no meio do tronco (Figura 6b). Para o diâmetro do lume das fibras, ocorreu variação significativa apenas no topo do tronco, sendo os maiores valores encontrados na região da casca (Figura 6c). A espessura da parede das fibras não variou no meio do tronco, e na base o menor valor ocorreu na medula, não diferindo entre as outras três posições radiais. No topo, a menor espessura foi notada na posição intermediária 2 , sem diferença significativa nas demais posições (Figura 6d).

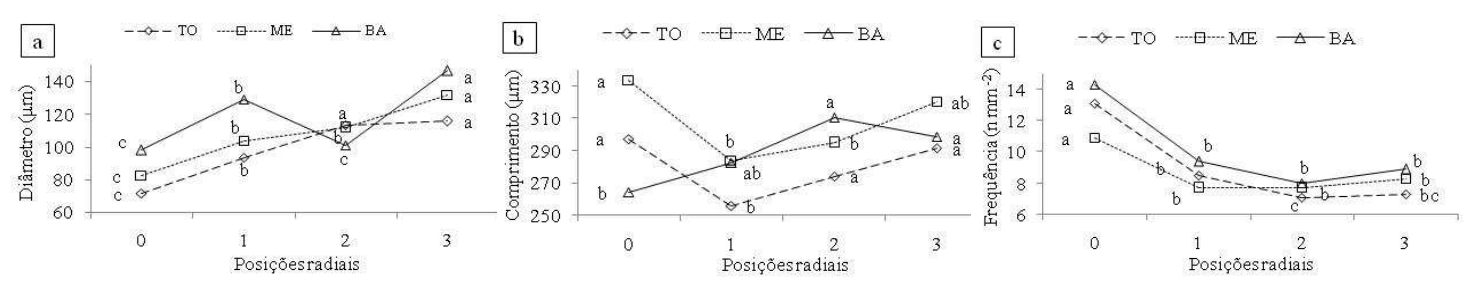

Figura 3. Variação radial dos vasos. a. Diâmetro dos vasos. b. Comprimentos dos elementos de vaso. c. Frequência dos vasos. Letras distintas indicam diferenças significativas entre as posições radiais: medula (0), intermediária 1 (1), intermediária 2 (2) e casca (3) em cada altura: base (BA), meio (ME) e topo (TO). $\mathrm{P}<0,05$ pelo teste de Tukey.

Figure 3. Radial variation of the vessels. a. Vessel diameter. b. Vessel element length. c. Vessel frequency. Different letters indicate significant differences between the radial positions: pith (0), intermediate 1 (1), intermediate 2 (2) and bark (3) at each height: base (BA), middle (ME) and top (TO). $\mathrm{P}<0.05$ by Tukey test.
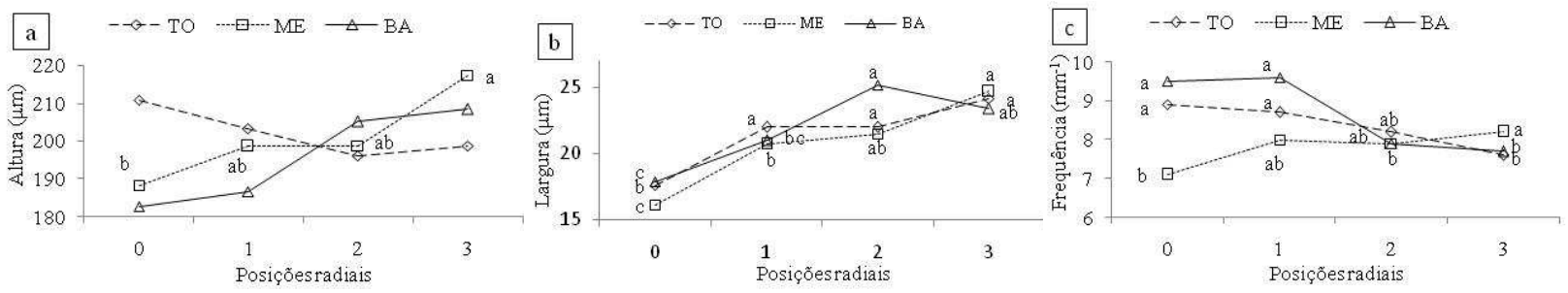

Figura 4. Variação radial dos raios. a. Altura dos raios. b. Largura dos raios. c. Frequência dos raios. Letras distintas indicam diferenças significativas entre as posições radiais: medula (0), intermediária 1 (1), intermediária 2 (2) e casca (3) em cada altura: base (BA), meio (ME) e topo (TO). $\mathrm{P}<0,05$ pelo teste de Tukey.

Figure 4. Radial variation of the rays. a. Rays height. b. Rays width. c. Rays frequency. Different letters indicate significant differences between the radial positions: pith (0), intermediate 1 (1), intermediate 2 (2) and bark (3) at each height: base (BA), middle (ME) and top (TO). P <0.05 by Tukey test.

\section{Propriedades}

$\mathrm{Na}$ base do tronco, a densidade aparente foi maior na posição intermediária 1 . No meio do tronco, o maior valor ocorreu na posição intermediária 2. No topo do tronco, os dois maiores valores foram observados nas posições intermediárias 1 e 2 (Figura 7a).

A velocidade de propagação do som (v) mostrou variação diferente conforme a altura da árvore. $\mathrm{Na}$ base do tronco foi mais alta na casca; no meio do tronco, a menor v ocorreu na posição intermediária 2 e no topo o menor valor foi notado na medula (Figura $7 b$ ).

Para o módulo de elasticidade dinâmico (MOEd), na base do tronco, o menor valor ocorreu na medula. No meio do tronco, a posição intermediária 1 mostrou o maior MOEd, enquanto que no topo do tronco, os dois maiores valores ocorreram nas duas posições intermediárias (Figura 7c). 

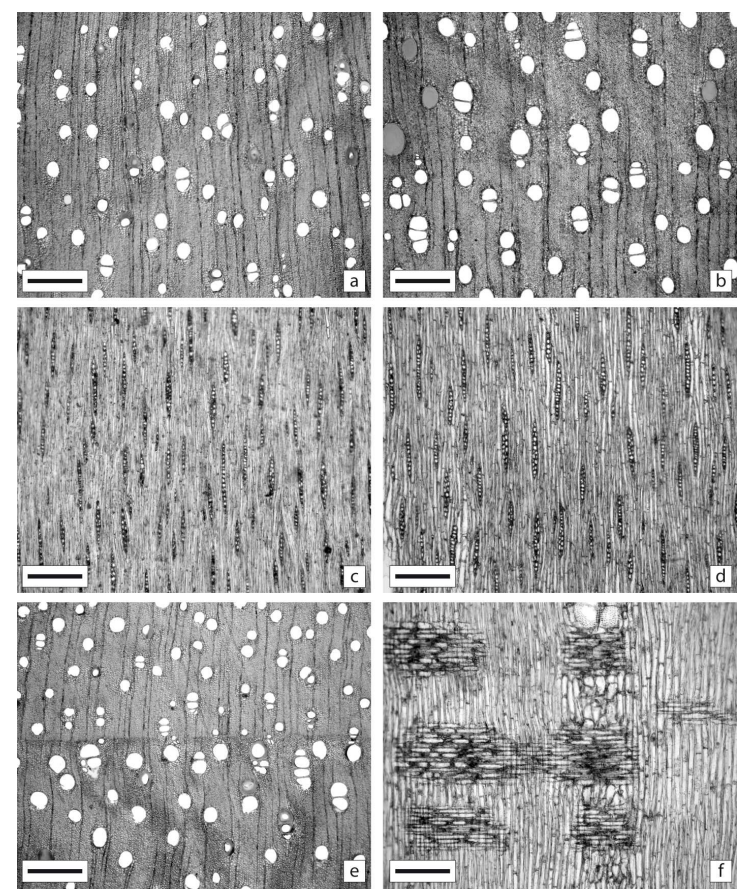

Figura 5. Lenho de Plathymenia reticulata. a-b. Secções transversais da medula e casca, respectivamente, evidenciando a diferença no diâmetro dos vasos. c-d. Secções tangenciais da medula e casca (notar raios mais largos na casca d). e. Secção transversal com visualização do marcador da camada de crescimento. f. Secção radial mostrando os raios com a presença de conteúdo. Figuras a, b, e barra de escala $=500 \mu \mathrm{m}$. Figuras c, d, f barra de escala $=200 \mu \mathrm{m}$.

Figure 5. Plathymenia reticulata wood. a-b. Transverse sections of pith and bark, respectively, demonstrating the difference in vessel diameter. c-d. Tangential sections of pith and bark (note wider rays in the bark d). e. Transverse section with growth layer. f. Radial section showing rays with content. Figures a, b, e scale bar $=500 \mu \mathrm{m}$. Figures c, d, f scale bar $=200 \mu \mathrm{m}$.
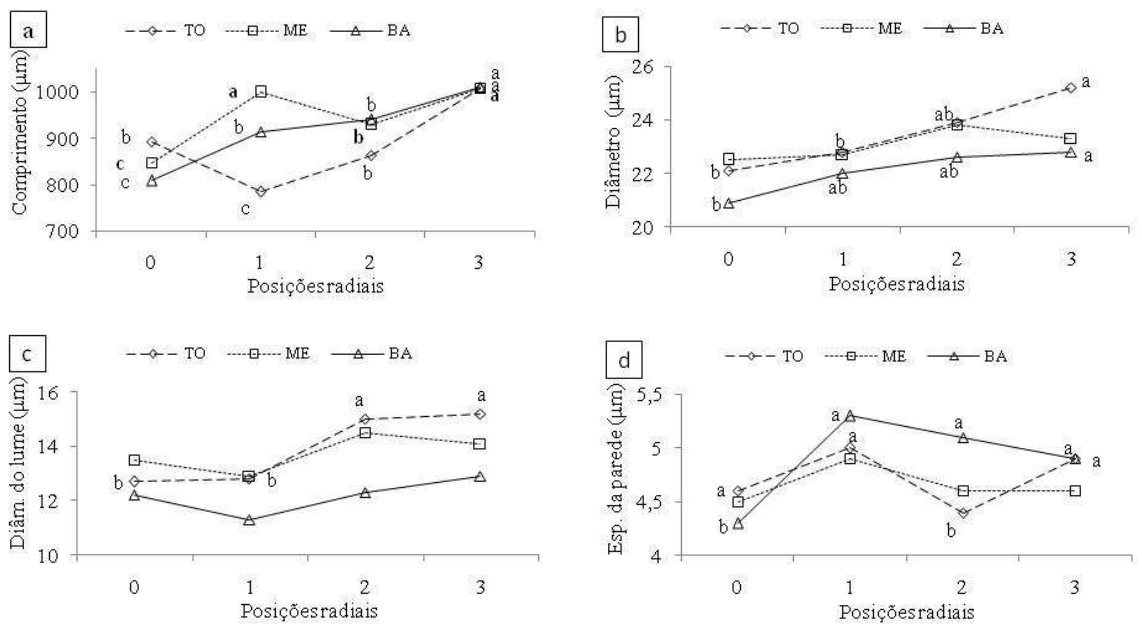

Figura 6. Variação radial das fibras. a. Comprimento das fibras. b. Diâmetro das fibras. c. Diâmetro do lume das fibras. d. Espessura da parede das fibras. Para as abreviações, ver figura 3. P<0,05 pelo teste de Tukey.

Figure 6. Radial variation of the fibers. a. Fibers lenght. b. Fiber diameter. c. Fiber lumen diameter. d. Fiber wall thickness. For abbreviations, see figure 3. P $<0.05$ by Tukey test. 

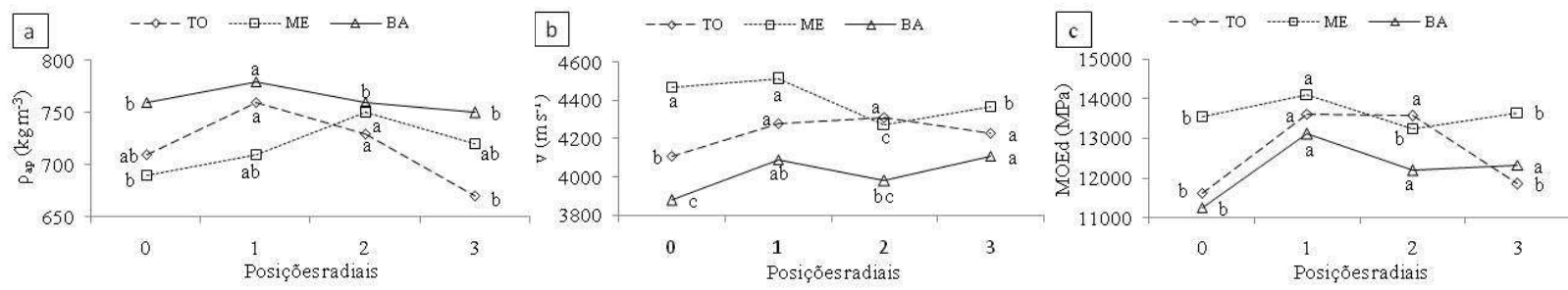

Figura 7. Variação radial das propriedades. a. Densidade aparente $\left(\rho_{a p}\right)$. b. Velocidade de propagação do som (v). c. Módulo de elasticidade dinâmico (MOEd). Para abreviações, ver figura 3. P <0,05 pelo teste de Tukey.

Figure 7. Radial variation of the properties. a. Specific gravity $\left(\rho_{a p}\right)$. b. Speed of sound propagation (v). c. Dynamic modulus of elasticity (MOEd). For abbreviations, see figure 3. $\mathrm{P}<0.05$ by Tukey test.

\section{Relação entre anatomia e propriedades}

Por meio das análises de correlação de Pearson, identificou-se relação positiva entre a densidade aparente e a frequência de raios, além de relações negativas entre a densidade aparente e o lume das fibras, entre a velocidade de propagação do som e a frequência dos raios e entre o módulo de elasticidade dinâmico e a frequência dos vasos (Tabela 2).

Tabela 2. Coeficiente de correlação de Pearson $(r)$ e grau de significância $(p)$ entre as características anatômicas e a densidade aparente, velocidade de propagação do som e módulo de elasticidade dinâmico na madeira de Plathymenia reticulata.

Table 2. Pearson correlation coefficient $(r)$ and significance level $(p)$ among the anatomical features and specific gravity, speed of sound propagation and dynamic modulus of elasticity in Plathymenia reticulata wood.

\begin{tabular}{lcccccccccc}
\hline \multirow{2}{*}{ Propriedades } & \multicolumn{10}{c}{ Características anatômicas } \\
\cline { 2 - 12 } & DV & CV & FV & AR & LR & FR & CF & DF & LF & PF \\
\hline$\rho_{\text {ap }}$ & & & & & & & & & & \\
$r$ & $-0,265$ & $-0,436$ & 0,088 & $-0,157$ & 0,143 & $\mathbf{0 , 6 0 3}$ & $-0,320$ & $-0,522$ & $\mathbf{- 0 , 6 2 4}$ & 0,311 \\
$p$ & 0,405 & 0,157 & 0,784 & 0,627 & 0,658 & $\mathbf{0 , 0 3 8}$ & 0,310 & 0,081 & $\mathbf{0 , 0 3 0}$ & 0,325 \\
$\mathbf{v}$ & & & & & & & & & & \\
$r$ & 0,235 & 0,294 & $-0,480$ & 0,154 & $-0,062$ & $\mathbf{- 0 , 5 6 3}$ & 0,196 & 0,449 & 0,495 & $-0,105$ \\
$p$ & 0,463 & 0,354 & 0,114 & 0,634 & 0,846 & $\mathbf{0 , 0 4 9}$ & 0,540 & 0,143 & 0,102 & 0,746 \\
MOE $_{\mathrm{d}}$ & & & & & & & & & & \\
$r$ & 0,134 & 0,101 & $\mathbf{- 0 , 5 8 6}$ & 0,073 & 0,094 & $-0,289$ & 0,050 & 0,239 & 0,206 & 0,115 \\
$p$ & 0,678 & 0,754 & $\mathbf{0 , 0 4 5}$ & 0,820 & 0,769 & 0,362 & 0,876 & 0,454 & 0,521 & 0,722 \\
\hline
\end{tabular}

DV: diâmetro dos vasos; CV: comprimento dos elementos de vaso; FV: frequência de vasos; AR: altura dos raios; LR: largura dos raios; FR: frequência dos raios; CF: comprimento das fibras; DF: diâmetro das fibras; LF: lume das fibras; PF: espessura da parede das fibras; $\rho_{a p}$ : densidade aparente; v: velocidade de propagação do som; $\mathrm{MOE}_{\mathrm{d}}$ : módulo de elasticidade dinâmico. As relações significativas estão destacadas em negrito.

\section{DISCUSSÃO}

De maneira geral, em $P$. reticulata a variação radial das características dos vasos e fibras mostrou um padrão comum para diversas espécies, ou seja, aumento no diâmetro dos vasos e comprimento das fibras e diminuição na frequência dos vasos em direção da casca, nas três alturas. Esse padrão reflete as alterações entre madeira juvenil e adulta, que são influenciadas pela maturidade do câmbio (TSOUMIS, 1968). Vários autores encontraram o mesmo padrão em espécies exóticas e nativas. Em um estudo semelhante, Urbinati et al. (2003) estudaram a variação radial e axial em Terminalia ivorensis, uma espécie de origem africana e conhecida popularmente no Brasil como sete-copas. Os autores investigaram material em três diferentes alturas $(1,3 ; 11,3$ e $21,3 \mathrm{~m})$, e para os vasos reportaram aumento no diâmetro e no comprimento do elemento na direção da casca nas três alturas analisadas, 
enquanto que a frequência de vasos diminuiu na mesma direção, com certa diferença entre as alturas. Fan et al. (2009), em quatro espécies da família Fagaceae, também observaram aumento no diâmetro dos vasos na direção da casca. Esse mesmo padrão também foi reportado em espécies nativas, como nos estudos de Lima et al. (2010) em Croton floribundus e Longui et al. (2009) em Luehea divaricata.

O aumento no diâmetro dos vasos da medula para a casca pode proporcionar aumento no transporte de água, o que é importante, considerando que, conforme a árvore cresce, há um aumento da biomassa e consequentemente maior número de folhas e células que devem receber água e nutrientes. Assim, vasos de maior diâmetro são mais eficientes na condução hidráulica, o que pode aumentar a capacidade de fotossíntese (CARLQUIST, 2001). Essa afirmação é reforçada pela lei de HagenPoiseuille, que avalia a dinâmica de fluidos através de tubos cilíndricos. Tyree et al. (1994) exemplificam essa lei para o fluxo de água através dos vasos do lenho, que também podem ser tratados como tubos. De acordo com os autores, um vaso com $40 \mu \mathrm{m}$ de diâmetro apresenta a mesma condutância de 16 vasos com $20 \mu \mathrm{m}$ de diâmetro ou 256 vasos com $10 \mu \mathrm{m}$ de diâmetro.

Como mencionado anteriormente, as posições axiais não foram analisadas estatisticamente. Contudo, é possível inferir sobre a variação das características dos vasos e o "tradeoff" entre eficiência hidráulica ou resistência mecânica. Com exceção da posição intermediária 2 , o diâmetro dos vasos diminuiu da base para o topo do tronco nas outras três posições radiais. A frequência dos vasos mostrou valores mais altos na base do tronco, oscilando nas duas outras posições radiais. Esse resultado pode indicar um ajuste na condução de água da base para o tronco, no qual se nota um aumento na segurança, uma vez que, de acordo com Zimmermann (1983), vasos de menor diâmetro são mais seguros, porém menos eficientes na condução de água. Além disso, o resultado também pode se relacionar com a resistência da madeira. Baas et al. (2004) mencionaram relação negativa entre o diâmetro ou a frequência de vasos com a resistência à ruptura. Assim, infere-se que regiões do tronco com menor frequência e diâmetro dos vasos sejam mais resistentes quando comparadas àquelas com maior número ou com vasos de maior diâmetro.

As diferenças nos raios são pouco exploradas nos trabalhos de variação radial, contudo, entendese que as alterações nessa característica sejam importantes no armazenamento e mobilização de carboidratos. Em $P$. reticulata, a altura dos raios apresentou variação significativa apenas no meio do tronco, com aumento em direção da casca. Já a largura dos raios aumentou em direção da casca nas três alturas. Ocorreu diminuição na frequência dos raios na base e no topo, o que pode estar relacionado com a redução do número de iniciais radiais e com o aumento do número de iniciais fusiformes, consequentemente, com aumento na proporção de fibras (URBINATI et al., 2003). De maneira geral, no presente estudo notou-se um aumento no volume dos raios em direção da casca. Tal resultado pode contribuir para o transporte radial periódico de carboidratos que são mobilizados para a reativação do câmbio (COSTA et al., 2006). Infere-se que, à medida que a árvore fica mais velha e com maior porte, o aumento no volume dos raios pode fornecer a quantidade adequada de açúcares para a retomada de crescimento após um período de dormência do câmbio.

Em regiões tropicais, a disponibilidade hídrica promove a sazonalidade cambial, sendo que uma estação seca anual com dois a três meses com precipitação menor que $60 \mathrm{~mm}$ interfere no funcionamento do câmbio e induz a formação de camadas de crescimento (WORBES, 1995). A caracterização climática do município de Assis, área de coleta das amostras aqui estudadas, indica que os meses de julho e agosto historicamente apresentam precipitação menor do que $50 \mathrm{~mm}$, sendo, portanto propícios à formação das camadas. Além disso, nos meses de abril e agosto ocorre déficit hídrico, o que também pode contribuir na sazonalidade do câmbio.

Raios mais volumosos, comparando-se com raios de menor volume, permitem armazenar mais carboidratos no verão, período de maior síntese, para ocorrer mobilização em períodos de estresse ou na primavera seguinte. Como P. reticulata, segundo Durigan et al. (1997), perde todas suas folhas no inverno, sugere-se que a mobilização das reservas de carboidratos para manter a árvore viva é de extrema importância, até que novas folhas se desenvolvam e a copa esteja novamente formada para realizar fotossíntese e nova produção de açúcares. Segundo Kozlowski e Pallardy (1997), grande quantidade de amido é oxidada na respiração e outra parte acumulada como reserva nas células dos raios de forma abundante próximo do câmbio, diminuindo a quantidade nas partes mais internas do alburno e sendo quase inexistente no cerne. Ao analisar os raios próximos do câmbio e ao longo das posições radiais, não foram encontrados grãos de amido, o que sugere que os mesmos devem ter sido mobilizados para suprir 
as necessidades energéticas das plantas no período de estresse. Além disso, o amido armazenado nas células do parênquima radial e axial que estão em contato com os vasos pode desempenhar um papel importante na recuperação de vasos embolizados. Salleo et al. (2004) encontraram relação positiva entre a recuperação da perda de condutividade hidráulica e a hidrólise de amido, que, segundo os autores, foi muito evidente nas células parenquimáticas ao redor dos vasos.

As fibras desempenham papel fundamental na sustentação das árvores. Além disso, variações nessas células têm sido empregadas para diferenciar lenho juvenil de adulto (GATTO et al., 2008; TSUCHIYA; FURUKAWA, 2009). Em P. reticulata, mesmo com algumas flutuações, observou-se aumento no comprimento das fibras da medula para a casca nas três alturas, sendo esta, entre as características das fibras, a que aparentemente melhor indicou as alterações ao longo do amadurecimento do câmbio, uma vez que os valores obtidos próximos à casca foram significativamente maiores do que aqueles da medula nas três alturas.

Entre as diferentes alturas, notou-se um aumento na espessura da parede das fibras entre a medula e a casca, estatisticamente comprovada na base do tronco, no qual estão representadas todas as camadas de crescimento das árvores. Além disso, na base, com exceção da medula, as paredes foram mais espessas do que no meio e topo do tronco. Tal resultado pode refletir um ajuste estrutural das árvores para melhorar a sustentação, pois fibras de paredes mais espessas podem contribuir no aumento da resistência, uma vez que a base do tronco é forçada a suportar todo o peso da árvore quando esta é balançada sob a ação do vento, ainda mais nos períodos em que os frutos desenvolvem-se e o peso da copa aumenta. A ação do vento sobre a fisiologia e anatomia das árvores tem sido explorada em diversos trabalhos e discutida em termos de tensão, deformação e resistência da madeira. A deformação do tecido pode ser elástica ou plástica. No primeiro caso, as alterações são reversíveis e a estrutura celular retorna à configuração original após a deformação. Já quando há uma deformação plástica, ocorre uma injúria no tecido e o indivíduo pode ou não se recuperar (TELEWSKI, 1995).

A densidade da madeira serve como um indicativo para estimar outras propriedades e relacionase com as dimensões e frequências das células (HOADLEY, 2000). Dessa forma, pode ser empregada para inferir a resistência mecânica e também a eficiência hidráulica do lenho. A relação positiva entre a densidade aparente e a frequência de raios é difícil de ser explicada, considerando que as células dos raios possuem paredes mais finas do que as das fibras e elementos de vasos. Assim, o resultado esperado seria uma relação negativa com a densidade da madeira, pois as células dos raios contribuiriam menos com a massa e consequentemente com a densidade. Contudo, a maior frequência dos raios ocorreu nas regiões mais internas do tronco, onde há maior quantidade de extrativos. Como em $P$. reticulata há distinção entre cerne e alburno, sugere-se que os extrativos presentes nas células dos raios podem ter contribuído para um aumento na densidade, o que explicaria em parte a relação positiva entre a densidade aparente e a frequência de raios. Bowyer et al. (2003) reportaram que madeiras com maior teor de extrativos têm a tendência de apresentar maior densidade. Outra provável explicação para esse resultado é que as variações no diâmetro e frequência dos vasos, bem como no diâmetro, espessura da parede e frequência das fibras possam compensar o aumento na frequência dos raios.

Fibras com maiores valores de lume estão relacionadas negativamente à densidade das amostras. Isso deve-se ao fato de o lume representar espaços vazios que não contribuem com a massa da madeira. Assim, salvo algumas exceções, as fibras são as células em maior proporção nas madeiras, o que explica a grande influência delas nas propriedades físico-mecânicas.

A velocidade de propagação do som na direção longitudinal é diretamente proporcional à elasticidade da madeira (SOCIETY OF WOOD SCIENCE AND TECHNOLOGY, 2009). Essa característica pode ser determinada por meio de ensaios não destrutivos e tem sido empregada na avaliação de descontinuidades, irregularidades e vazios que ocorrem naturalmente, como característica intrínseca da árvore ou de sua interação com o meio, e que afetam as propriedades mecânicas da madeira. $\mathrm{Na}$ literatura há vários trabalhos que indicam correlações significativas entre ensaios destrutivos e não destrutivos, relacionando positivamente a velocidade de propagação do som com o módulo de elasticidade (TSEHAYE et al., 2000; GONÇALEZ et al., 2001; MINÁ et al., 2004). No presente estudo, observou-se uma relação negativa entre a velocidade de propagação do som e a frequência dos raios. Considerando que a velocidade pode ser empregada para estimar o módulo de elasticidade e que este seja o quociente entre a tensão aplicada a um corpo e a deformação que ela provoca, sendo utilizado para explicar a relação entre a rigidez e a flexibilidade da madeira, quanto maior for o módulo de elasticidade, 
mais rígida é a madeira, apresentando menor deformação. Por outro lado, quanto menor o módulo de elasticidade, mais flexível é a madeira (GREEN et al., 1999). Dessa forma, regiões com maior frequência de raios podem apresentar maior flexibilidade e contribuir para equilibrar estruturalmente o tronco. Notar que a frequência dos raios aumentou no meio do fuste e diminuiu na base e topo.

A relação negativa entre o módulo de elasticidade dinâmico $\left(\mathrm{MOE}_{\mathrm{d}}\right)$ e a frequência de vasos na madeira de $P$. reticulata pode ser entendida da seguinte forma: quanto maior a frequência dos vasos, menor será a rigidez e consequentemente maior sua flexibilidade. Como a frequência de vasos diminui na direção da casca, pode-se inferir que nas regiões mais próximas da medula a madeira de $P$. reticulata seja mais flexível e possa apresentar maior encurvamento antes de romper-se. Esse resultado, além de apresentar relação com o uso da madeira, também pode ser discutido em um contexto ecológico, uma vez que a presença de madeira mais flexível nos primeiros anos de vida pode contribuir na resistência da árvore jovem, que pode vergar-se mais com a ação do vento sem quebrar, ao passo que uma árvore jovem que possua madeira com alta rigidez pode se quebrar mais facilmente sob a mesma intensidade de vento.

Além disso, como o módulo é um indicativo da rigidez e uma maior frequência de vasos pode aumentar a condutividade hidráulica, a relação negativa entre essas duas características pode indicar uma adaptação da planta, que alterna regiões com maior rigidez e menor condutividade hidráulica ou viceversa. A relação negativa entre essas características também foi observada por Christensen-Dalsgaard et al. (2008) em raízes tabulares de Tachigali melinonii e Xylopia nitida.

\section{CONCLUSÕES}

- O aumento do diâmetro dos vasos na direção da casca, determinado pela maturação do câmbio, influencia na eficiência hidráulica, pois vasos mais largos apresentam maior eficiência na condução de água. Por outro lado, maior frequência de vasos correlacionou-se negativamente com o MOEd (maior flexibilidade). Esse resultado, além de apresentar relação com o uso da madeira, também pode ter contribuído na resistência da árvore jovem à ação dos ventos, pois quanto maior a flexibilidade, o risco de ruptura é menor.

- O aumento na largura dos raios no sentido radial pode contribuir no armazenamento de amido, que é hidrolisado e os açúcares mobilizados em períodos de menor síntese. A hidrólise do amido também pode atuar na recuperação de vasos embolizados, o que representa uma estratégia importante em períodos com déficit hídrico e provável formação de bolhas de ar nos vasos.

- Regiões com raios mais frequentes diminuem a velocidade longitudinal de propagação do som. Como ela também pode ser usada como um indicativo do módulo, sugere-se que regiões com maior frequência de raios podem apresentar maior flexibilidade e contribuir para equilibrar estruturalmente o tronco.

- Nas amostras da base do fuste, com exceção da posição medula, as demais posições mostram fibras com paredes mais espessas do que no meio e topo do tronco. Tal resultado pode refletir um ajuste estrutural para melhorar a sustentação das árvores quando elas recebem uma carga extra proporcionada pelo vento ou pelo desenvolvimento de frutos. A relação negativa entre a densidade aparente e lume das fibras ocorreu, pois o lume representa espaços vazios que não contribuem na massa da madeira.

\section{AGRADECIMENTOS}

Os autores agradecem a Sônia Godói Campião, da Seção de Madeira e Produtos Florestais, e Daniela Araújo, graduanda em Agronomia da UNESP - Campus Ilha Solteira, pelo auxílio laboratorial, e também ao senhor Daniel Lombardi, na determinação da velocidade de propagação do som.

\section{REFERÊNCIAS}

BAAS, P.; EWERS, F. W.; DAVIS, S. D.; WHEELER, E. A. Evolution of xylem physiology. In: POOLE, I.; HEMSLEY, A. (eds.). Evolution of Plant Physiology. London: Elsevier Academic Press. (Linnaean Society Symposium Series), 2004. p. 273 - 295. 
BERLYN, G. P.; MIKSCHE, J. P. Botanical microtechnique and cytochemistry. Iowa: The Iowa University Press, 1976. 326 p.

BOWYER, J. L.; SHMUlSKY, R.; HAYGREen, J. G. Forest Products and Wood Science - An Introduction. 4. ed. Iowa State Press, A Blackwell Publishing Co. 2003.

CARLQUIST, S. Comparative wood anatomy: systematic, ecological and evolutionary aspects of dicotyledons wood. Springer Verlag, Berlin. 2001.

CARVALHO, P. E. R. Espécies arbóreas brasileiras. Brasília DF: Embrapa Informações Tecnológica; Colombo: Embrapa Floresta, 2008. v. 3.

CENTRO DE PESQUISAS METEOROLÓGICAS E CLIMÁTICAS APLICADAS À AGRICULTURA (CEPAGRI). Clima dos Municípios Paulistas. 2010 Disponível em: <http://www.cpa.unicamp.br/ outras-informacoes/clima-dos-municipios-paulistas.html>. Acesso em: 05/08/2010.

CHRISTENSEN-DALSGAARD, K. K.; ENNOS, R. A.; FOURNIER. M. Interrelations between hydraulic and mechanical stress adaptations in woody plants. Plant Signaling \& Behavior. v. 3:7, p. 463 - 465, 2008.

COSTA, C. G.; CALLADO, C. H.; CORADIN, V. T. R.; CARMELLO-GUERREIRO, S. M. Xilema. In APPEZZATO-DA-GLÓRIA, B.; CARMELLO-GUERREIRO, S. M. (orgs.). Anatomia Vegetal. 2. ed. Ed. UFV, Viçosa. 2006.

DURIGAN, G.; FIGLIOLIA, M. B.; KAWABATA, M.; GARRIDO, M. A. O.; BAITELlO, J. B. Sementes e mudas de árvores tropicais. São Paulo: Páginas e Letras, 1997. 65 p.

FAN, X.; CAO, K. F.; BECKER, P. Axial and radial variations in xylem anatomy of angiosperm and conifer trees in Yunnan, China. IAWA Journal, Leiden, v. 30, n. 1, p. 1 - 13, 2009.

GATTO, D. A.; HASElEIN, C. R.; SANTINI, E. J.; MARCHIORI, J. N. C.; DURLO, M. A.; CALEGARI, L.; STANGERLIN, D. M. Características tecnológicas das madeiras de Luehea divaricata, Carya illinoinensis e Platanus $x$ acerifolia quando submetidas ao vergamento. Ciência Florestal, Santa Maria, v. 18, n. 1, p. 121 - 131, 2008.

GONÇALEZ, J. C.; VALLE, A. T.; COSTA, A. F. Estimativas das constantes elásticas da madeira por meio de ondas ultrassonoras (ultrassom). Revista Cerne, Lavras. v. 7, n. 2, p. 65 - 80, 2001.

GREEN, D. W.; WINANDY, J. E.; KRETSCHANN, D. E. Mechanical properties of wood. In: WOODHANDBOOK: wood as an engineering material. Madison: Forest Products Laboratory, 1999. (Gen. Tech. Rep. 113: 1 - 46).

HOADLEY, B. Understanding Wood: A Craftsman's Guide to Wood Technology. Taunton Press Inc,. 2nd Revised edition, 2000.

IAWA COMMITTEE. List microscope features of hardwood identification. IAWA Bulletin, Leiden, v. 10, n. 3, p. 221 - 259, 1989.

JENRICH, H. Vegetação arbórea e arbustiva nos altiplanos das Chapadas do Piauí Central: características, ocorrência e empregos. Teresina: GTZ, 1989.

KOZLOWSKI, T. T.; PALlARDY, S. G. Physiology of Woody Plants. Academic Press, New York, 1997.

LIMA, I. L.; ZANON, B. R.; LONGUI, E. L.; FLORSHEIM, S. M. B. Variação radial da densidade básica e dimensões celulares de Croton floribundus. Floresta, Curitiba, v. 40, p. 663 - 670, 2010.

LONGUI, E. L.; LIMA, I. L.; FLORSHEIM, S. M. B.; BUFOLO, A. Variação anatômica radial do lenho de açoita-cavalo (Luehea divaricata) e sua influência na densidade aparente. Revista do Instituto Florestal, v. 21, n. 2, p. 181 - 190, 2009.

LUCCHI, G. The Use of Empirical and Scientific Methods to Measure the Velocity of Propagation of Sound. J. Violin Soc. New York. v. 9/1, p. 107 - 123, 1986. 
MAX, J. C. M.; MELO, A. C. G.; HONDA, E. A.; DURIGAN, G.; MALÍCIA, L. C.; SOUZA, M. B. M.; CARDOSO, M. M.; BÔAS, O. V.; RAMOS, V. S.; CONTIÉRI. W. A. Plano de manejo da Floresta Estadual de Assis. IF Sér. Reg., São Paulo, n. 30, p. 1 - 80, jun, 2007.

MINÁ, A. J. S.; OLIVEIRA, F. G. R.; CALIL JR., C.; DIAS, A. A.; SALES, A. Avaliação não destrutiva de postes de madeira por meio de ultrassom. Scientia Florestalis. v. 65, p. 188 - 196. 2004.

SALlEO, S.; LOGUllO, M. A.; TRIFILO, P.; NARDINI, A. New evidence for a role of vesselassociated cells and phloem in the rapid xylem refilling of cavitated stems of Laurus nobilis L. Plant, Cell and Environment. v. 27, p. 1065 - 1076. 2004.

SANDRONI, P. Novíssimo dicionário de economia. 10. ed. São Paulo: Best Seller, 2002.

SASS, J. E. Botanical microtechniche. Ames: The Iowa State College Press, 1951. 326 p.

SILVEIRA, V.; ROSADO, S. C. S.; TRUGILHO, P. F.; CARVALHO, D.; MARTINS, S. C. Variação dimensional da madeira de clones de Eucalyptus cultivados em diferentes espaçamentos. Cerne, Lavras, v. 5, n. 2, p. 105 - 124, 1999.

SOCIETY OF WOOD SCIENCE AND TECHNOLOGY. Properties of Wood. Disponível em: <http://www.swst.org/teach/teach2/properties2.pdf>. Acesso em: 11/08/2010.

TELEWSKI, F. W. Wind-induced physiological and developmental responses in trees. In: Coutts MP, Grace J, editors. Wind and Trees. Cambridge: Cambridge University Press. 1995.

TSEHAYE, A.; BUCHANAN, A. H.; WALKER, J. C. F. Sorting of logs using acoustics. Journal of Wood Science \& Technology. v. 34, p. 337 - 344, 2000.

TSOUMIS, G. Wood as raw material. Pergamon Press, Oxford. 1968.

TSUCHIYA, R.; FURUKAWA, I. Radial variation of vessel lumen diameter in relation to stem increment in 30 hardwood species. IAWA Journal. v. 30, n. 3, p. 331 - 342, 2009.

TYREE, M. T.; DAVIS, S. D.; GOCHARD, H. Biophysical perspectives of xylem evolution: is there a tradeoff of hydraulic efficiency for vulnerability to dysfunction? IAWA Journal. v. 15, p. 335 - 360, 1994.

URBINATI, C.; AZEVEDO, A. A.; SILVA, E. A. M.; LISBOA, P. L. Variação estrutural quantitativa no lenho de Terminalia ivorensis A. CHEV, Combretaceae. Acta Botanica Brasilica, São Paulo, v. 17, n. 3, 421 - 437, 2003.

WAGNER, K. R.; EWERS, F. W.; DAVIS, S. D. Tradeoffs between hydraulic efficiency and mechanical strength in the stems of four co-occurring species of chaparral shrubs. Oecologia v. 117, p. 53 - 62, 1998.

WOODRUM, C. L.; EWERS, F. W.; TELEWSKI, F. W. Hydraulic, biomechanical, and anatomical interactions of xylem from five species of Acer (Aceraceae). American Journal of Botany. v. 90, n. 5, p. $693-699,2003$.

WORBES, M. How to measure growth dynamics in tropical trees - a review. IAWA Journal. v. 16, n. 4, p. $337-351,1995$.

ZIMMERMANN, M. H. Xylem structure and the ascent of sap. New York: Springer-Verlag. 143 p. 1983. 